\title{
MONTE CARLO SIMULATIONS OF VESICLES AND FLUID MEMBRANES TRANSFORMATIONS
}

\author{
STEFANO PIOTTO ${ }^{1 *}$ and FABIO MAVELLI ${ }^{2}$ \\ ${ }^{1}$ Institute for Inorganic Chemistry, ETH Hoenggerberg, Wolfgang-Pauli-Strasse 10, \\ CH-8093 Zürich, Switzerland \\ ${ }^{2}$ Dipartimento di Chimica, University of Bari, Via Orabona 4, 70125 Bari, Italy \\ (*author for correspondence, e-mail: piotto@inorg.chem.ethz.ch)
}

(Received 20 October 2002; accepted in revised form 19 March 2003)

\begin{abstract}
The appearance of compartmentalization is recognized as a key step in biogenesis. The study of the dynamical behaviour of amphiphilic close membranes at equilibrium or under some external stress (osmotic pressure or dehydration process) can be useful in order to better elucidate the role of vesicles in the origin of life and to get insight into the molecular and membrane properties that bring to a spontaneous vesicle division. A Monte Carlo approach to simulate the evolution of close membranes under an external stress will be presented. This approach is mainly based on the accepted surface energy model introduced by Helfrich (1973) and Seifert (1997a). Some preliminary results will be also illustrated and possible developments and limits of this method discussed.
\end{abstract}

Keywords: Monte Carlo, origin of life, simulation, vesicle

\section{Introduction}

The appearance of compartmentalization has been claimed by different authors (Deamer and Oro', 1980; Goldrach, 1958; Morowitz et al., 1988), as a key step in biogenesis: the continuum of chemical processes that led from an essentially random mixture of organic and inorganic molecules to the first assemblies recognizable as living systems. The presence of a boundary that separates the inside from the outside appears to be per se fundamental to individuate a living organism (Fleischhaker, 1990) since all known life forms are cellular and each cell is separated from the essentially aqueous outside environment by a thin bilayer water-insoluble membrane composed of both lipid and protein (Singer and Nicolson, 1972). Then, from the knowledge of contemporary living organisms it seems only reasonable to include the self-assembly of amphiphilic molecules into bilayer membranes as an essential step in the evolution of life on earth.

In most experimental conditions, amphiphilic molecules can self-assemble spontaneously into spherical closed bilayered shells with only a small amount of external work (for instance mild shaking) (Small, 1986). These aggregates are called vesicles and they consist of a water pool encapsulated into a bilayer membrane of lipids. They have flexible surfaces and can show a huge variety of morphologies and topologies depending on the material properties and on the 
environment physicochemical parameters. Possible routes that bring from simple molecules to the synthesis of amphiphiles able to form vesicles have been hypothesized (Ourisson and Nakatani, 1999) and experimental works that show evolutionary autocatalytic pathways from surfactant precursor molecules to this aggregates in presumable prebiotic environments have also been published by Luisi and coworkers (Bachmann, 1992; Blöchliger, 1998; Wick, 1995). Dehydrationhydration cycles have been also proposed (Deamer and Barchfeld, 1982) as mechanisms for the encapsulation of macromolecules or ions inside vesicle: during drying vesicles flatten and fuse to form lamellar structures and then, when rehydration occurs, lamellae swell and disperse into large vesicles. Finally, experimental conditions where a vesicle ancestor can split into two daughter aggregates with the same topology have been reported (Döbereiner et al., 1993; Wick et al., 1995). In this framework, the study of the dynamical behaviour of amphiphilic membranes at equilibrium or under some external stress (osmotic pressure increase due to a dehydration process) can be useful in order to better elucidate the role of membranes in the origin of life and to get insights into the molecular and membrane properties that bring to a spontaneous vesicle fission, i.e. without involving complex proteins as in modern cells.

\section{Surface Energy of Lipidic Membranes}

Helfrich was the first to point out the role of curvature in the description of vesicle conformation (Helfrich, 1973) by expressing the surface energy of a flexible membrane in terms of the mean curvature $(\mathrm{H})$, and Gaussian curvature $(\mathrm{K})$ :

$$
E \equiv \frac{\kappa}{2} \oint_{\text {surface }}\left(2 H-c_{0}\right)^{2} d A+\kappa_{G} \oint_{\text {surface }} K d A,
$$

where $A$ is the surface area, $c_{0}$ is the spontaneous curvature and $\kappa$ and $\kappa_{G}$ are the bending rigidity and the Gaussian bending rigidity respectively. The mean curvature and the Gaussian curvature at a point on a surface are the average and the product of the principal curvatures at that point, the principal curvatures being the minimum and maximum curvatures of curves on the surface lying in planes including the tangent vector at the given point. A spontaneous curvature $c_{0}$ can arise either from a different chemical composition of the two membrane layers, or from different environments on both sides of the bilayer.

The surface energy together with constraints on the total area (assuming a fixed number of lipids) and the enclosed volume (assuming no osmotic stress) defines the spontaneous-curvature (SC) model. Therefore, neglecting effects due to convection and thermal fluctuations, vesicles will acquire the shape that minimises the surface energy at given constrains.

Seifert extended the theoretical work of Helfrich introducing the difference between inner and outer layer areas (Döbereiner et al., 1997; Seifert, 1997a, b). 
During years, several works have been then published where Euler-Lagrange equations were analytically solved to find the best shape in quasi-equilibrium vesicular systems under specific constrains (Kralj-Iglic et al., 1999; Seifert, 1997a; Seifert and Wintz, 1996). One limitation of this approach is that it cannot handle systems under strong stressing conditions, such as high osmotic pressure or long-range deformation and, furthermore, the temporal evolution of membranes cannot be addressed, but only minimum energy membrane conformations can be determined.

To follow the time evolution of many-particle systems, Molecular Dynamics (MD) is normally used. This method is based on Newton dynamics and semi-empirical force-fields that specify inter-molecular force laws. Although this approach can provide atomic detailed descriptions of lipid membranes and it can reproduces local bilayer properties (Goetz and Lipowsky, 1998; Lindahl and Edholm, 2000; Tieleman et al., 1997), it is computationally very expensive to be feasible for studying a vesicle as a whole (Ben-Shaul, 1996; Tieleman et al., 1997) since a very large amount of interacting molecules has to be taken into account. Another problem is that MD can permit the investigation of the system time evolution in the range of nanoseconds (Tieleman et al., 1997) that is a very short time scale compared to vesicle conformation changes.

Recently, another possibility for studying the conformations of lipid vesicles has been developed. By using a triangularized surface approach, the lipidic membrane is represented at a mesoscopic level as a collection of vertices of connected triangles and the curvature energy is minimized numerically by the gradient descent method (Brakke, 1992) or by using a Monte Carlo approach (Brakke, 1992; Koibuchi and Yamada, 2000a, b). Koibuchi and Yamada adopted a simplified Polyakov's rigid string model (Polyakov, 1986) that worked out to be very efficient in analyzing the dynamics of flexible membranes (Brakke, 1992; Koibuchi and Yamada, 2000a, b). Furthermore, these authors introduced an approximate formula to estimate the energy of triangularized surfaces that is computationally less expensive than Equation (1). The surface energy was split into two contributions:

$$
E=c_{1} E_{1}+c_{2} E_{2},
$$

where the first term, called the area energy, depends on the size of the surface, whereas the second one estimates the bending energy, $c_{1}$ and $c_{2}$ being two adjustable parameters. Two different pairs of expressions have then been proposed and tested to estimate these two energetic contributions: the first based on the triangle sides (edge):

$$
\begin{aligned}
& E_{1}=\sum_{\text {surface }} \text { edge }^{2} \\
& E_{2}=\sum_{\text {edge }}\left(1-\cos \vartheta_{\text {edge }}\right)
\end{aligned}
$$


and the second one, based on the triangle areas $\left(A_{t r}\right)$ :

$$
\begin{aligned}
& E_{1}=\sum_{\text {surface }} A_{t r} \\
& E_{2}=\sum_{\text {surface }} \frac{1}{A_{t r}} \sum_{i=1}^{3} \operatorname{edge}^{2}\left(1-\cos \vartheta_{\text {edge }}\right)
\end{aligned}
$$

$\theta$ being the angle between the normal lines of two adjacent triangles. It is possible to demonstrate that the three formulae of the surface energy, Equations (1) and (2) along with the definitions (3)-(6), are equivalent for an implemented triangularization (Koibuchi and Yamada, 2000a).

Following this idea, we have developed a computer program based on a Monte Carlo algorithm to simulate the time evolution of a close membrane that undergoes conformation changes in order to minimise the surface energy under physical perturbations. In the next section, this program and the parameters needed to run in silico experiments of vesicle transformations will be presented.

\section{Setting Up In Silico Experiments}

The use of a triangular mesh rather than a smooth and continuous surface permits to reduce the calculation of geometrical properties, as for instance volume and surface, into an easy and automatic task (Piotto, 2000). A triangulated surface (trSurface) can be considered a suitable representation if surface integrals, analytically calculated on the continuous surface, are equal to summations over all triangles within the expected accuracy. It should be clear that increasing the number of used triangles can improve the calculation accuracy at the cost of increasing the computational time. Working on a triangularized mesh Gaussian $(K)$ and mean $(H)$ curvature can be also easily estimated (Piotto, 2000) and consequently the bilayer surface energy can be evaluated and numerically minimised accordingly Helfrich's (Helfrich, 1973) or the Koibuchi's (Koibuchi and Yamada, 2000b). Hamiltonians, as reported in Equations (1) and (2)-(6), respectively.

The iterative MC procedure used to find the minimal energy conformation of a given structure is the traditional Metropolis Method (Metropolis et al., 1953). It simply consists in randomly changing the state of the system, i.e. the position of some vertices of the triangularized surface, and storing the new conformation if its energy $\left(E_{2}\right)$ is less than before $\left(E_{1}\right)$. When the energy results are to be higher, the new conformation will be accepted or rejected if an acceptance probability law $\left(P=\exp \left[-\left(E_{2}-E_{1}\right) / k_{B} T\right]\right)$ is randomly satisfied, where $T$ is temperature and $k_{B}$ the Boltzman's constant. The acceptance condition is verified if generating a pseudo-random number $u$, uniformly distributed between $0-1$, will result $u<P$. Whereas a Monte Carlo procedure can be computationally very expensive to converge to the minimal energy conformation, an obvious advantage is its robustness 
and the wide range of membrane transformations that can encompass. Given a trSusface oscillating around the surface energy minimum conformation, an external perturbation can be simulated opportunely modifying the expression of the surface energy. In the case of an external osmotic stress an additive energetic contribution $E_{V}$ has to be considered in the definition of energy:

$$
E \equiv \frac{\kappa}{2} \oint_{\text {surface }}\left(2 H-c_{0}\right)^{2} d A+\kappa_{G} \oint_{\text {surface }} K d A+E_{v}
$$

or

$$
E=c_{1} E_{1}+c_{2} E_{2}+E_{V} .
$$

According to Seifert (Seifert, 1997a), this osmotic energetic term can be approximated as follows:

$$
E_{v} \approx \frac{R T c V_{0}}{2}\left(\frac{V}{V_{0}}-1\right)^{2},
$$

where $R$ is the gas constant, $V$ is the volume of the vesicle and $c$ is the concentration of molecules caught within the vesicle. Subscript 0 indicates the equilibrium value.

It is worthwhile to mention that the second term on the right side of Equation (7) is constant for a given topology and then it can be neglected for any vesicle transformation at constant genus (Seifert, 1997a).

The osmotic perturbation can be introduced by suddenly setting $V_{0} \ll V$ (osmotic shock) or more smoothly imposing a linear decrease of $V_{0}$ with time (slow dehydration). The energy minimization can be then performed setting different constrains, for instance keeping the vesicle surface $A$ constant along with the spontaneous curvature $c_{0}$ and/or the reduced volume that is the ratio between the vesicle volume and the volume of a sphere of equal surface. In Figure 1 a diagram of some possible in silico experiments of vesicular evolution at constant topology is illustrated.

To perform all these kind of simulations we developed a C++ object-oriented computer program called EM-solv. This program has been compiled and linked to the AVS/Express graphical libraries that supply all the necessary graphic tools for the real time visualization of the simulations. Triangularized surfaces of any topology can be easily imported from input data files or automatically generated by using a friendly graphical user interface. A wide variety of geometrical surface properties can also be calculated and it is possible to set parameters to simulate stress conditions and analyse the MC evolution of the system in real time. Moreover, EM-solv can export simulation outcomes in the most common graphic data file formats and produce compressed videos as well. All the algorithms and the data structures implemented in EM-solv have been designed and optimized for the simulation of flexible membranes (Piotto and Mavelli, 2003). EM-solv has been 


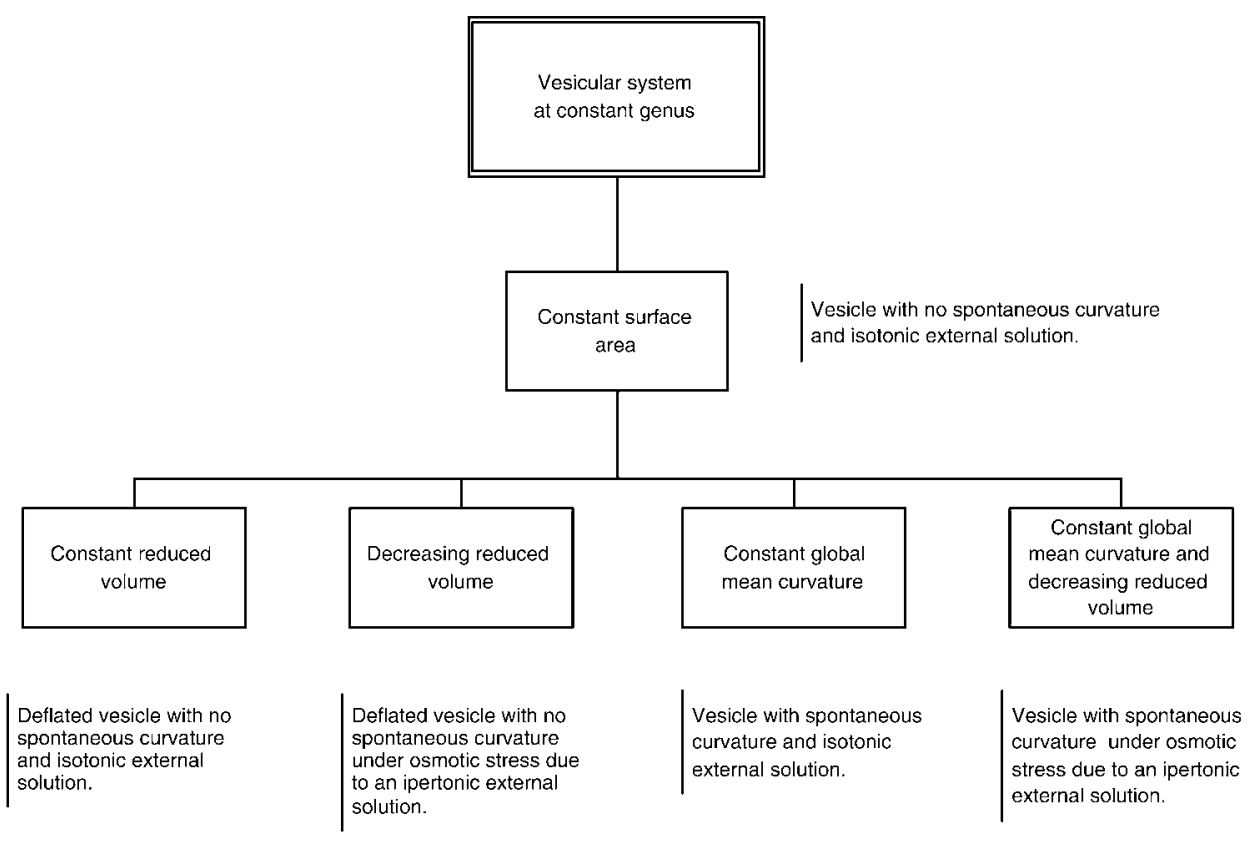

Figure 1. Scheme of the in silico experiments: different simulation types reported along with the required constrains and physical meanings.

firstly tested on a set of transformations with fixed topology in order to validate the method and to evaluate the best set of parameters to investigate dynamics and division of vesicles in prebiotic conditions. All the parameters needed to start a simulation are listed in Table I. It should be stressed that running in silico experiments requires the same attentions of test-tube experiments, since only an accurate choice of parameters can lead to successful results. It is worth to mention that the symmetry of the initial triangularized vesicle is critical in determining the convergence time of the algorithm. Whereas a nonsymmetrical vesicle converge to the conformation corresponding to a minimum of energy in tens of iterations, a high symmetrical surface (sphere like) does not converge even after thousands of iterations.

\section{Results}

Herein the attention will be focused on a spherical vesicle that undergoes an osmotic perturbation, i.e. a fixed topology transformation with the only constrains of constant surface. The adopted surface energy will be the Koibuchi and Yamada's formula defined by Equations (8), (5), (6), and (9). Simulation results obtained using Equation (7) will be not illustrated, since they are substantially in agreement with those shown. The target systems are vesicles of POPC (palmitoyl-oleoyl- 


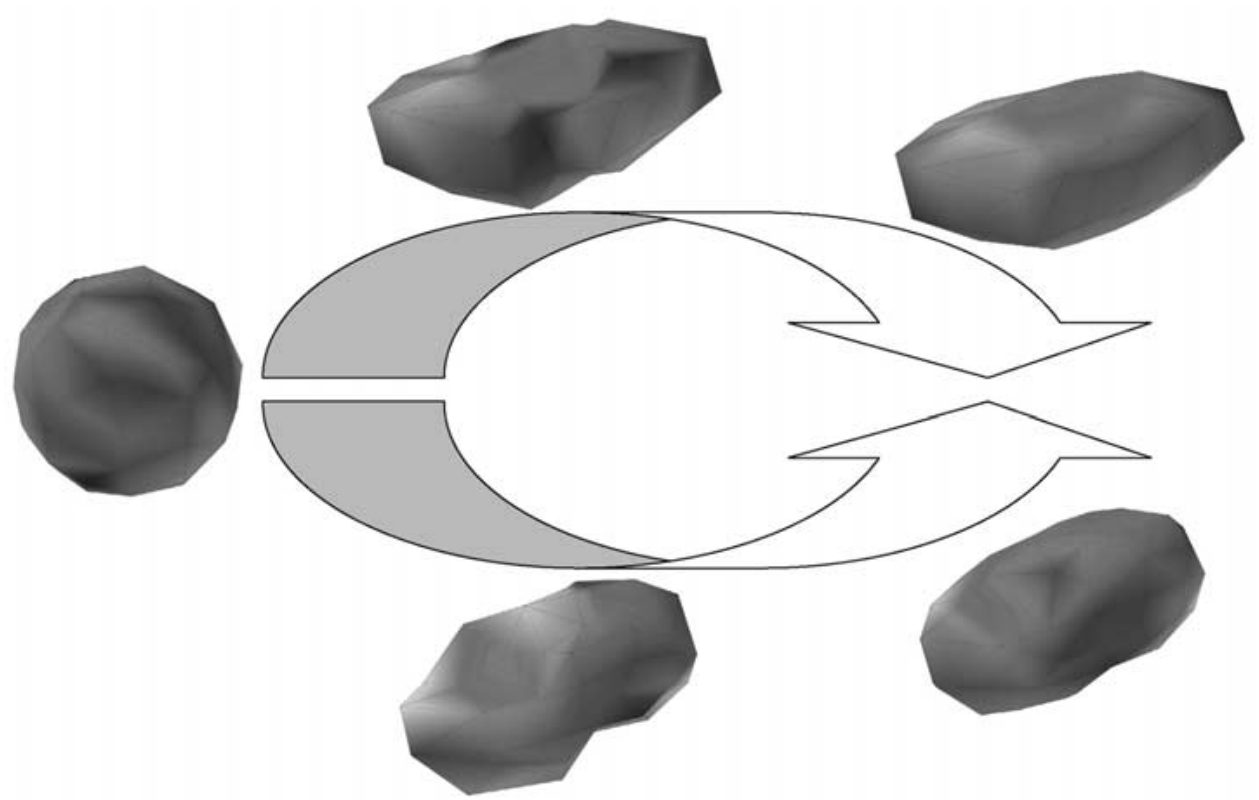

Figure 2. Relaxation of a $200 \mathrm{~nm}$ diameter vesicle undergoing an external osmotic stress. A smooth variation of the external aqueous ionic strength corresponds to a smooth deflation of the triangular vesicle, on top. The case of an osmotic shock is shown on bottom. The colour map of the surface mean curvature shows the roughness difference between the two cases.

phosphatidylcholine), and oleic acid/oleate, since they have been extensively studied (Blöchliger, 1998; Wick et al., 1995) as prototypes of minimal cells. The most remarkable aspect of the simulation is that the time evolution of triangularized vesicles accurately represents the experimentally observed dynamics of real vesicular systems such as oleate/oleic acid giant vesicles (Blöchliger, 1998; Wick et al., 1995). In fact, spheres or ellipsoids can only roughly approximate real vesicles because, even if these shapes correspond to the optimal equilibrium structures, thermal fluctuations and convective motions continuously perturb them. Moreover, the osmotic energy term, Equation (9), can strongly perturb the vesicle geometry because of the different energy scale of the osmotic and the surface contributions (Seifert, 1997a). When the osmotic term $E_{v}$ in Equation (8) vanishes the energy area $E_{1}$ and the bending energy $E_{2}$ are responsible for the smoothness of the membrane, vice versa in presence of an osmotic stress a variety of morphologies can appear since, in some cases, $E_{v}$ can overcome of some orders of magnitude the first two contributions. Consequently the smoothness of the membrane is not guaranteed and geometrically highly stressed conformations can appear. Figure 2 shows two relaxation paths of a vesicle of approximately $200 \mathrm{~nm}$ in diameter undergoing an osmotic shock (far from equilibrium process), on bottom, or a gently osmotic stress (quasi-equilibrium process), that simulates a slow dehydration process, on top. 


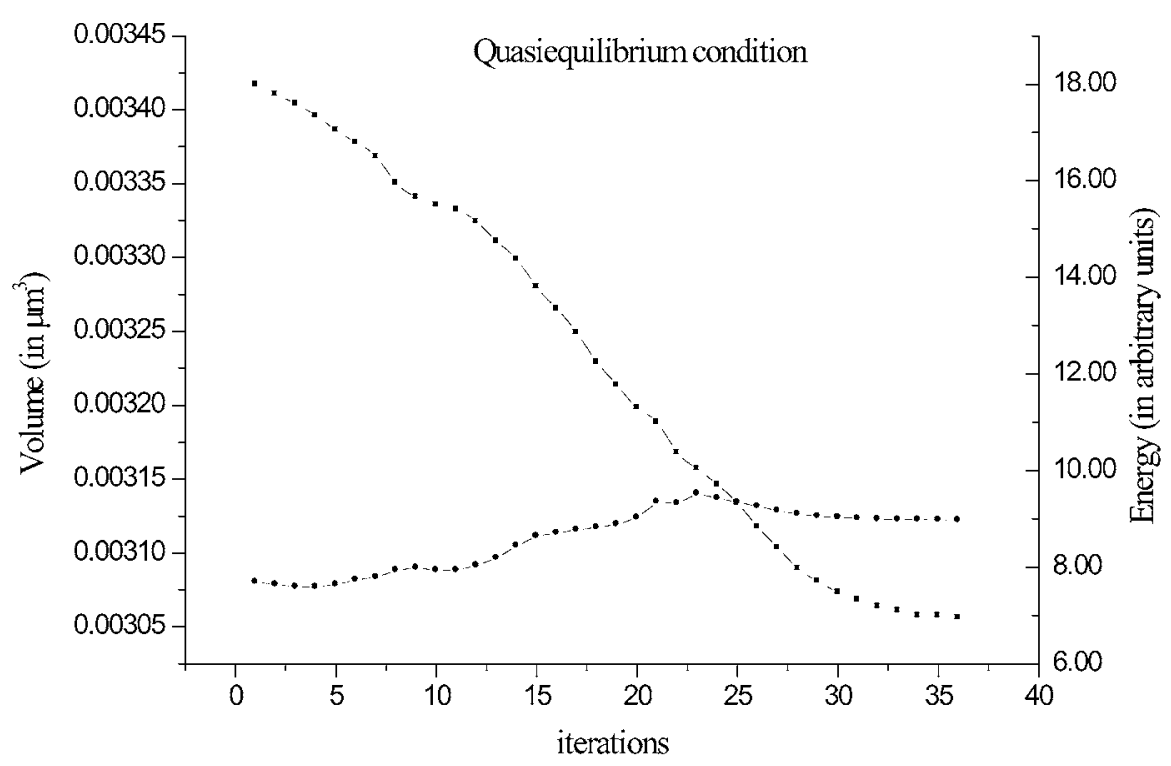

Figure $3 a$. Surface Energy and Volume time evolution of a $200 \mathrm{~nm}$ diameter vesicle undergoing to a gently osmotic stress: quasi-equilibrium process.

The surface energy and volume diagrams versus time are reported in Figures $3 a, b$.

When the osmotic energy is comparable with the area and the bending energy the vesicle remains always in a quasi equilibrium state: its surface energy increases slowly as a consequence of the osmotic stress and conversely its volume decreases regularly up to the equilibrium value, see Figure 3a. Throughout the time evolution the vesicle does not show high curvature regions. These results confirm the supposed large molecule encapsulation mechanism (Deamer and Barchfeld, 1982) based on dehydration-hydration cycles showing as during a slow dehydration vesicles tend to flatten.

On the other hand, if the osmotic energy is much larger than the area and the bending energy (osmotic shock), the vesicle deflates rapidly (see Figure 3b) and the membrane appears rough and inhomogeneous. In this region, conformations with high bending energy appear, and in some cases higher genus structures can be formed. In these conditions, the vesicle fission process can occur. Moreover, if the osmotic perturbation exceeds the mechanical limits of the membrane, the vesicle can collapse.

\section{Conclusions}

Monte Carlo simulations of triangulated vesicles worked out to be an extremely powerful tool to investigate vesicle transformations. The main achievement of this 


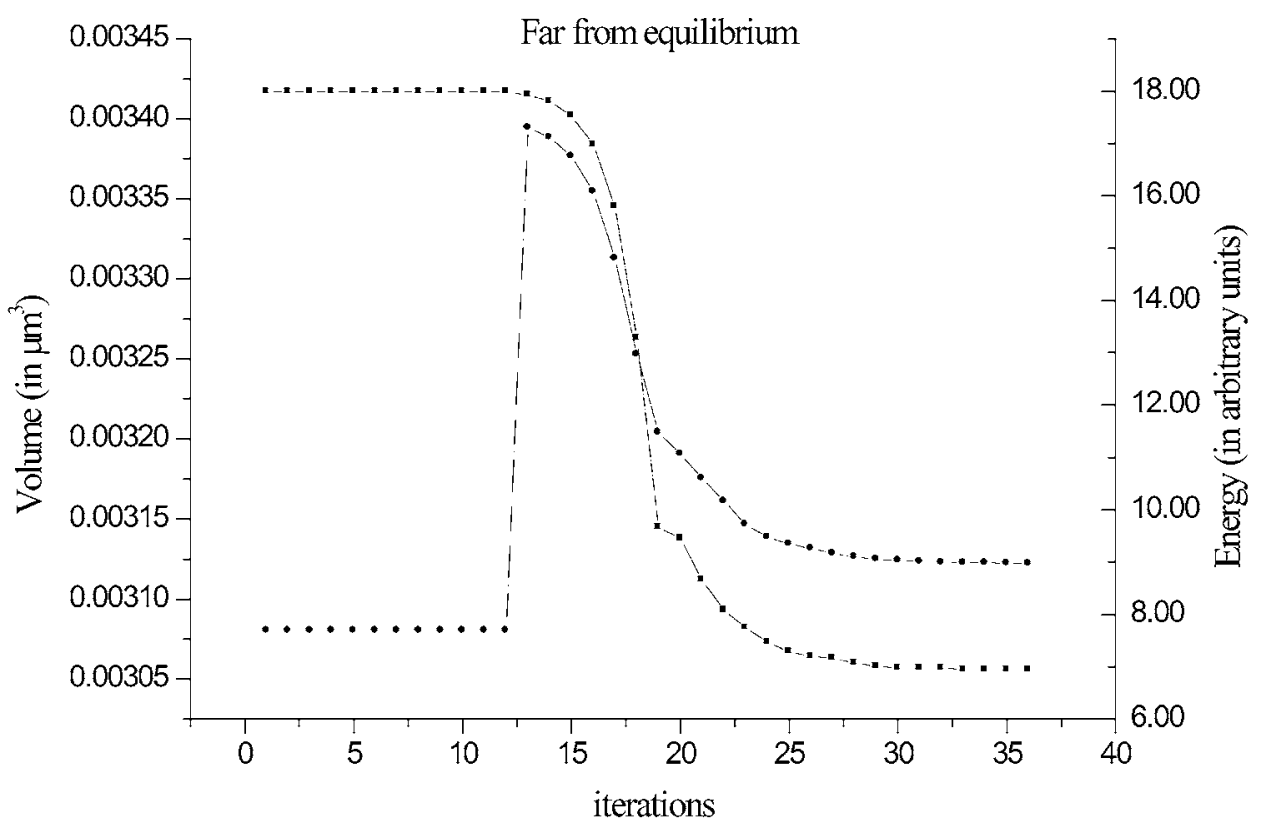

Figure $3 b$. Surface Energy and Volume time evolution of a $200 \mathrm{~nm}$ diameter vesicle undergoing an osmotic shock: far from equilibrium process.

approach is to account for the hugely different behaviour of vesicles in quasiequilibrium states or in far from equilibrium conditions. In the first case vesicle dynamics consists in fluctuations around a minimal energy conformation and the simulation results are in good qualitative agreement with the analytical approach due to Helfrich and Seifert. On the other hand, far from equilibrium processes can be also studied by means of MC simulations, and the formation of high bending energy structures can be also elucidated as a consequence of a strong perturbation. In the present work we illustrated a simulation of lipid vesicles undergoing an osmotic stress due to a slow or a quick evaporation, in order to simulate the behaviour of vesicles in a prebiotic water pool. The method can be extended to analyse transformations of membranes with varying spontaneous curvature $c_{0}$ and vesicular aggregates with different topology.

Furthermore, the knowledge of surfactant molecular properties can be used to parameterize triangulated membranes, and consequently to help the design of vesicular systems composed of mixed amphiphiles capable to show complex cellular behaviours, like divisions, channels formation, and invaginations, triggered by simple chemical-physical perturbations. 


\section{References}

Bachmann, P. A., Luisi, P. L. and Lang, J.: 1992, Autocatalytic Self-Replication Micelles as Model for Prebiotic Structures, Nature 357, 57-59.

Ben-Shaul, A.: 1996, The 'New' Science of 'Complex fluid', J. Phys. Chem 100.

Blöchliger, E., Blocher, M., Walde, P. and Luisi, P. L.: 1998, Matrix Effect in the Size Distribution of Fatty Acid Vesicles, J. Phys. Chem. B 102, 10383-10390.

Brakke, K. A.: 1992, The Surface Evolver, Experimental Mathematics 1 (2), 141-165.

Deamer, D. W. and Barchfeld, G. L.: 1982, Encapsulation of Macromolecules by Lipid Vesicles under Simulated Prebiotic Conditions, J. Mol. Evol. 18, 203-206.

Deamer, D. W. and Oro', J.: 1980, Role of Lipids in Prebiotic Structures, BioSystems 12, 167-175.

Döbereiner, H.-G., Evans, E., Kraus, M., Seifert, U. and Wortis, M.: 1997, Mapping Vesicle Shapes into the Phase Diagram: A Comparison of Experiment and Theory, Physical Review 55 (4), 44584474.

Döbereiner, H.-G., Kas, J., Noppl, D., Sprenger, I. and Sackmann, E.: 1993, Budding and Fission of Vesicles, Biophys. J. 65 (4), 1396-1403.

Fleischhaker, G. R.: 1990, Origins of Life: An Operational Definition, Orig. Life Evol. Biosphere 20, 127-137.

Goetz, R. and Lipowsky, R.: 1998, Computer Simulations of Bilayer Membranes: Self-Assembly and Interfacial Tension, Journal of Chemical Physics 108(17), 7397-7409.

Goldrach, R. J.: 1958. Surface Films: Their Collapse on Compression, the Shape and Size of Cells, and the Origin of Life, Surface Phenomena in Biology and Chemistry, Pergamon Press, New York.

Helfrich, W.: 1973, Elastic Properties of Lipid Bilayers: Theory and Possible Experiments, $Z$. Naturforsch. [C] 28 (11), 693-703.

Koibuchi, H. and Yamada, M.: 2000a, Phase Transition of a Model of Crystalline Membrane, International Journal of Modern Physics C 11 (3), 1509-1518.

Koibuchi, H. and Yamada, M.: 2000b, Phase Transition of a Model of Fluid Membrane, International Journal of Modern Physics C 11 (3), 441-450.

Kralj-Iglic, V., Heinrich, V., Svetina, S. and Zeks, B.: 1999, Free Energy of Closed Membrane with Anisotropic Inclusions, Eur. Phys. J. B 10, 5-8.

Lindahl, E. and Edholm, O.: 2000, Mesoscopic Undulations and Thickness Fluctuations in Lipid Bilayers from Molecular Dynamics Simulations, Biophysical Journal 79 (1), 426-433.

Metropolis, N., Rosenbluth, A. W., Rosenbluth, M. N., Teller, A. H. and Teller, E.: 1953, J. Chem. Phys. 21, 1087.

Morowitz, H. J., Heinz, B. and Deamer, D. W.: 1988, The Chemical Logic of a Minimum Protocell, Orig. Life Evol. Biosphere 18, 281-287.

Ourisson, G. and Nakatani, Y.: 1999, Addendum, Tetrahedron 55 (11), 3183-3190.

Piotto, S. P.: 2000. A Novel Surface Approach to Treat and Analyse Membranes, Micelles, Vesicles and their Transitions, Swiss Federal Institute of Technology, Zuerich.

Piotto, S. P. and Mavelli, F.: 2003, Monte Carlo Simulation of Curved Flexible Membranes, in preparation.

Polyakov, A. M.: 1986, Fine Structure of Strings, Nucl. Phys. B 268, 406.

Seifert, U.: 1997a, Configurations of Fluid Membranes and Vesicles, Advances in Physics 46(1), 13-137.

Seifert, U.: 1997b, Dynamics of Giant Vesicles, Mol. Cryst. Liq. Cryst. 292, 213-225.

Seifert, U. and Wintz, W.: 1996, Starfish Vesicles, Europhysics Letters 33 (5), 403-408.

Singer, S. J. and Nicolson, G. L.: 1972, The Fluid Mosaic Model of the Structure of Cell Membranes, Science 175, 720-731.

Small, D. M.: 1986, The Physical Chemistry of Lipids, Plenum Press, New York. 
Tieleman, D. P., Marrink, S. J. and Berendsen, H. J. C.: 1997, A Computer Perspective of Membranes: Molecular Dynamics Studies of Lipid Bilayer Systems, Biochimica et Biophysica Acta (BBA) - Reviews on Biomembranes 1331 (3), 235-270.

Wick, R., Walde, P. and Luisi, P. L.: 1995, Autocatalysis and Self-Reproduction of Giant Vesicles, J. Am. Chem. Soc. 117, 1435-1436.

Wick R., Walde, P. and Luisi P. L.: 1995, Autocatalysis and Self-Reproduction of Giant Vesicles, J. Am. Chem. Soc. 117, 1435-1436. 